\title{
TRIPLE COLLISIONS IN THE ISOSCELES 3-BODY PROBLEM
}

\author{
BY ERNESTO A. LACOMBA ${ }^{1}$ AND LUCETTE LOSCO
}

We consider here the plane 3-body problem of celestial mechanics forming an isosceles configuration at all times. We first study the topology of the energy submanifolds $E_{h}$ with the triple collision manifold $T$ (Mc Gehee [4]) as an added boundary, which corresponds to blowing up the collision. By a time transformation we scale the vectorfield extending it to the boundary. We then analyze the fictitious flow on $T$ to get information about the actual neighboring flow on $E_{h}$. Our results are akin to Mc Gehee's for the collinear case; but the fictitious flow is more interesting here, having 6 instead of 2 critical points.

Devaney [1] has simultaneously studied the flow of this problem. There is considerable overlap of his paper with the results we state after Theorem 1, except for his last section as explained at the end of this announcement. In [7] Simó has recently described the flow more completely.

To get such an isosceles motion, the two masses at the symmetrical vertices must be equal, with a fixed symmetry axis, about which the initial velocities must be balanced.

Denote by $\mu$ the equal masses and by $m$ the third one, introducing Jacobicoordinates [5]: $x \geqslant 0$ is the semidistance between the equal masses, and $y \in R$ is the signed distance from $m$ to the segment joining the others. Dividing out by a $2 \mu$ factor, we take the simplified lagrangian

$$
L(x, y, \dot{x}, \dot{y})=\left(\dot{x}^{2}+\dot{y}^{2} / \alpha^{2}\right) / 2+U(x, y),
$$

where $\alpha^{2}=1+2 \mu / m$, and the potential function is

$$
U(x, y)=\mu /(4 x)+m / \sqrt{x^{2}+y^{2}} .
$$

The associated hamiltonian (total energy) is

$$
E\left(x, y, p_{1}, p_{2}\right)=\left(p_{1}^{2}+\alpha^{2} p_{2}^{2}\right) / 2-U(x, y) \text {. }
$$

If we set $q=(x, y), p=\left(p_{1}, p_{2}\right)$ and define a matrix $M=\operatorname{diag}\left(1, \alpha^{-2}\right)$, the Hamilton equations for (3) can be writen in the familiar form

$$
\dot{q}=M^{-1} p, \quad \dot{p}=\operatorname{grad} U(q),
$$

and the energy relation $E=h$, defining any energy surface can be written as

$$
1 / 2 M^{-1} p^{2}=U(q)+h
$$

Received by the editors July 30, 1979 and, in revised form, February 14, 1980. AMS (MOS) subject classifications (1970). Primary 58F05, 70F05; Secondary $58 \mathrm{~F} 15$.

1 Research partially supported by CONACYT (Mexico), grant PNCB 170. 
By a coordinate and time transformation $q=\rho Q, p=\rho^{-1 / 2} P, d t=$ $\rho^{3 / 2} d \tau$ where $\rho^{2}=I=x^{2}+(y / \alpha)^{2}$ is the moment of inertia of the system, we get [4]:

$$
\begin{aligned}
& \rho^{\prime}=\lambda \rho, \quad \lambda=P \cdot Q, \\
& Q^{\prime}=M^{-1} P-\lambda Q, \quad M Q^{2}=1, \\
& P^{\prime}=\operatorname{grad} U(Q)+(\lambda / 2) P,
\end{aligned}
$$

while the energy relation (4), becomes

$$
1 / 2 M^{-1} P^{2}=U(Q)+\rho h .
$$

In (5) and (6), $\rho=0$ is not a singularity anymore, but defines the collision submanifold and its fictitious flow, independently of $h$. From (2) we see that $U$ still has a (regularizable) singularity in its first term, corresponding to a double collision of the equal masses.

In our case, the configuration $q$-space is the half-plane $x \geqslant 0$ with its origin as a triple collision, and the nonzero components of the $y$ axis as double collisions. Off collisions, (4) describes the energy surfaces as pinched circle bundles [8] over the subset $U(x, y) \geqslant-h$ in the half-plane $x>0$. We think of the Mc Gehee transformation as blowing up the origin to a small semicircle of radius $\epsilon>0$ (chosen so as not to intersect $U(x, y)=-h$ ). So, we have to add the condition $|q| \geqslant \epsilon$.

At the remaining points of the $y$ axis we have to regularize double collisions, which amounts to replacing the fibers by open real intervals, since only the value of $p_{2}$ (or of $\dot{y}$ ) distinguishes different collisions. We get the following result.

THEOREM 1. The topology of the regularized energy surfaces $E_{h}$ with triple collision boundary, is equivalent to the set

(a) $\left\{(x, y, z) \in R^{3}: 1<x^{2}+y^{2}+z^{2} \leqslant 4, x^{2}+y^{2} \neq 0, y^{2}+z^{2} \neq 0\right\}$, if $h \geqslant 0$,

(b) $\left\{(x, y, z) \in R^{3}: x^{2}+y^{2}+z^{2} \leqslant 4,(x \pm 1)^{2}+y^{2}+z^{2}>1 / 4, x= \pm 1 \Rightarrow\right.$ $y \neq 0\}$, if $h<0$.

This topology is the same as in the collinear 3-body problem and completes the topology of [4], where one focused on the triple collision boundary $\left(S^{2}-4\right.$ points).

To study the flow on the regularized collision manifold $T$ natural coordinates for equation (5) are $\rho, \varphi, \kappa, \psi$ where $Q=(\cos \varphi, \alpha \sin \varphi), \kappa^{2}=M^{-1} P^{2}, P=$ $\left(\kappa \cos \theta, \kappa \alpha^{-1} \sin \theta\right)$ and $\psi=\theta-\varphi ;-\pi / 2<\varphi<\pi / 2,-\pi \leqslant \psi \leqslant \pi$ :

$\rho^{\prime}=\rho \kappa \cos \psi$,

$\varphi^{\prime}=\kappa \sin \psi$,

$\kappa^{\prime}=-\mu \cos (\varphi+\psi) \sec ^{2} \varphi / 4-m / r^{3}\left[\cos \psi+\left(\alpha^{2}-1\right) \sin \varphi \sin (\varphi+\psi)\right]$

$$
+\kappa^{2} \cos \psi / 2 \text {, }
$$

$$
\kappa \psi^{\prime}=\mu \sin (\varphi-\psi) \sec ^{2} \varphi / 4-m / r^{3}\left[\sin \psi+\left(\alpha^{2}-1\right) \sin \varphi \cos (\varphi+\psi)\right]
$$


and where we set $r^{2}=|Q|^{2}$. The energy relation (6) becomes

$$
1 / 2 \kappa^{2}=\mu \sec \varphi / 4+m / r+\rho h .
$$

In these coordinates, the easiest way to get the critical points of (7) is by letting $\rho^{\prime}=\varphi^{\prime}=0$ and $d F / d \varphi=0$, where $F(\varphi)=\mu \sec \varphi / 4+m / r$ is the potential energy. The first two conditions give $\rho=0$ (the manifold $T$ ), $\sin \psi=0$, while $F$ has the local maximum $\varphi=0$. The two minima are given by

$$
\cos \varphi=r(\varphi) / 2 \text {. }
$$

If $\varphi_{0}$ is the positive solution to (9), we conclude that there are six critical points as shown in the figure below, corresponding by couples to the (Euler) collinear central configuration for $(0,0) \&(\pi, 0)$, and the two possible (Lagrange) equilateral configurations for $\left(0, \pm \varphi_{0}\right) \&\left(\pi, \pm \varphi_{0}\right)$ at the triple collision.

By a straightforward computation of the equations of variation, we get the following. Let $\delta, \delta^{\prime}= \pm 1$ from now on.

THEOREM 2. The eigenvalues of the vectorfield at the 6 hyperbolic critical points, are the following

\begin{tabular}{lcc}
\hline Points & $(\pi / 2+\delta \pi / 2,0) \quad$ Euler & $\left(\pi / 2+\delta \pi / 2, \delta^{\prime} \varphi_{0}\right)$ Lagrange \\
Eigenvalues & $\delta \kappa / 4 \pm 1 / 4 \sqrt{\kappa^{2}-28 \mu},-\delta \kappa$ & $\delta \kappa / 4 \pm \kappa\left(\sqrt{\left.19-18 / \alpha^{2}\right) / 4,-\delta \kappa}\right.$ \\
Character at $T$ & sink or source & saddles
\end{tabular}

where $\kappa^{2}=m\left(\alpha^{2}+7\right) / 4$ at Euler points and $\kappa^{2}=m\left(3+\alpha^{2}\right)^{3 / 2} /(4 \alpha)$ at Lagrange points.

Since $\mu>0, \alpha>1$, there are no zero eigenvalues as asserted. Each point has two eigenvalues whose real parts have different sign, and all of them are real at Lagrange points.

The above results are joint work of both authors; the following ones were obtained by the first author.

COROllaRY 1. Each hyperbolic point has an orbit and a two-dimensional invariant submanifold. For the Euler points the invariant submanifold is contained in $T$, while at Lagrange points the orbit is contained in $T$, and the submanifold is transversal.

We conclude that the flow on $T$ has saddles at Lagrange points, and sinks or sources at Euler points.

The figure below was drawn for the case where the masses satisfy $\alpha^{2}<$ $57 / 2$. Since there are nontrivial complex conjugate eignevalues, the flow actually spirals at Euler points. The dotted lines represent three possible routes for the remaining stable orbit at $\left(0, \varphi_{0}\right)$. Using numerical methods, Simó[7] has shown that the only case which does occur corresponds to the trajectory $A$ (starting 
asymptotically either from $(-\Pi, \Pi / 2)$, from the saddle $\left(-\Pi, \varphi_{0}\right)$ or from the source $(-\Pi, 0))$.

The edges $\psi= \pm \pi$ must be correspondingly identified. At top and bottom, regularization of double collisions amounts to identifying points $(\psi, \delta \pi / 2)$, with $(-\psi, \delta \pi / 2)$. This gives $S^{2}-4$ points for the topology of $T$, as required.

The actual triple collision orbits will be those approaching a critical point as $t \rightarrow+\infty$ or $t \rightarrow-\infty$ from outside $T$.

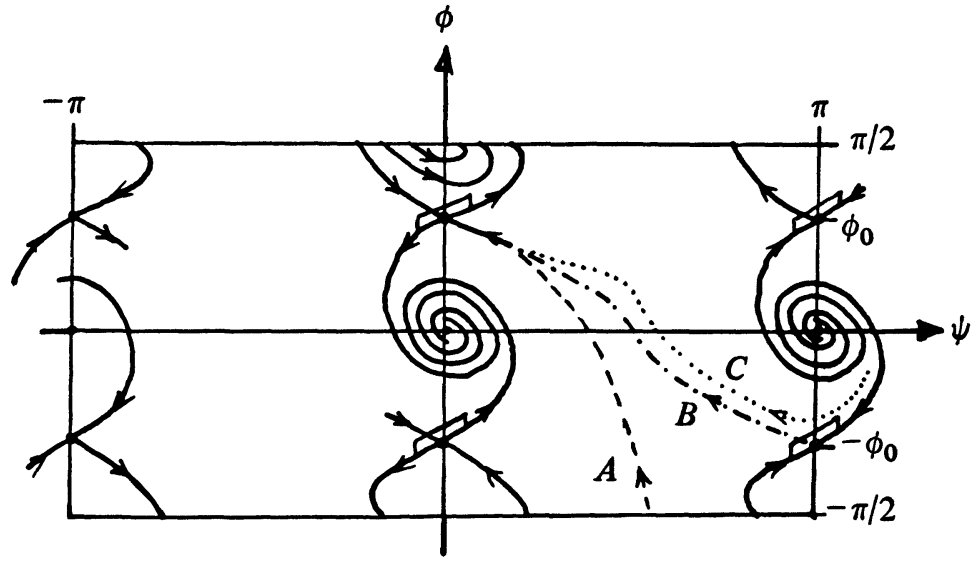

THEOREM 3. The triple collision orbits approaching Euler points are the invariant orbits coming to $T$. The set of triple collision orbits approaching Lagrange points are the invariant submanifolds.

THEOREM 4. The flow at $T$ is gradient-like with respect to the function $\lambda=\rho^{\prime} / \rho$.

Indeed from [3], we have $\lambda^{\prime}=1 / 2 M Q^{\prime 2} \geqslant 0$ when $\rho=0$, while $\lambda^{\prime}=0 \mathrm{im}$ plies $\lambda^{\prime \prime}=0, \lambda^{\prime \prime \prime}>0$ off critical points. This result makes sense of the fact that critical points of $F$ locate critical points of (7).

Using a similar technique, we can easily define an isolating block [2] for $T$. However, as a singularity, $T$ is not regularizable, with the possible exception of a discrete set of mass ratios where saddle connections appear in the above figure [6].

THEOREM 5. The coordinate $\rho$ is a hyperbolic Lyapunov function with regard to the regularized flow on each energy surface. Thus $\rho \leqslant \epsilon$ with $\epsilon>0$. small enough defines an isolating block for $T$.

Obviously, the behavior of orbits passing close to triple collision is more complicated than in [4]. For example, an orbit close to the stable manifold at $\left(\pi, \varphi_{0}\right)$ i.e., close to an equilateral configuration with $y>0$ may either end up approaching $(0,0)$ (a collinear configuration), or $(0, \pm \pi / 2)$, which means the equal masses come close together, with $y>0$ or $y<0$. The latter situation 
corresponds to the $m$-particle emerging with arbitrarily high velocity in one direction, while the $\mu$-particles emerge close together and move rapidly in the opposite direction, after the triple collision approach.

Devaney has made the behavior of the above orbits more precise, showing that they feature an arbitrarily large number of binary collisions while escaping. He also describes what he calls "billiard shots": orbits which in a first approach to collision behave as the orbits above, while on a second approach the binary pair separates rapidly and the third mass oscillates near their center of mass.

Based on a more complete picture of the flow on $T$ as described in the remark after Corollary 1 , Simó has made a finer description of the possibilities for close-collision orbits, and of ejection-collision orbits for negative energy, some of the latter ones actually turning out to be periodic.

\section{REFERENCES}

1. R. Devaney, Triple collision in the planar isosceles three body problem (to appear).

2. R. Easton, The topology of the regularized integral surfaces of the 3-body problem, J. Differential Equations 12 (1972), 361-384.

3. D. Eschbach, La collision triple du problème des trois corps, 3rd cycle doctoral thesis, Besançon, France, 1979.

4. R. Mc Gehee, Triple collision in the collinear 3-body problem, Invent. Math. 27 (1974), 191-227.

5. H. Pollard, Celestial mechanics, Carus Math. Monographs, no. 18, Math. Assoc. Amer., 1976. 21 (1980).

6. C. Simo, Masses for which triple collision is regularizable, Celestial Mechanics

7. - Analysis of triple collision in the isosceles problem (to appear).

8. S. Smale, Topology and mechanics. I, Invent. Math. 10 (1970), 305-331.

DEPARTMENT OF MATHEMATICS, UNIVERSIDAD AUTONOMA, METROPOLITANA-IZTAPALAPA, P. O. BOX 55-534, MEXICO 13, D. F.

DEPARTMENT OF THEORETICAL MECHANICS, UNIVERSITY OF BESANCON, 25030 BESANÇON, FRANCE 